\title{
Stress Development and Fracture of Surface Nucleated Cristobalite
} on Silica Glass

\author{
Ryan C. Breneman, ${ }^{\dagger} *$ and John W. Halloran** \\ Department of Material Science and Engineering, University of Michigan, Ann Arbor, MI
}

\begin{abstract}
Stress development and fracture of isolated cristobalite spherulites in amorphous silica matrix were observed. High purity bulk silica was annealed to produce partial surface crystallization consisting of isolated and impinged spherulites in an amorphous matrix. The stress state of the amorphous silica surrounding cristobalite spherulites was qualitatively examined using crossed-polars microscopy. Fracture was observed to occur with many spherulites encircled by cracks in the matrix and other spherulites observed to self-fracture in a "mudcracking" pattern. The fracture was found to be size dependent with encircling matrix cracks occurring as a minority phenomena in spherulites 20-70 microns in diameter and "mud-cracking" self-fracture to occur in all spherulites over 70 microns in diameter. The stresses develop as a result of the strain associated with the $4.9 \%$ volume reduction in the cristobalite on transitioning from beta-to-alpha phase at $\sim 250^{\circ} \mathrm{C}$. Observed fracture behaviors were modeled. Matrix cracks encircling spherulites were found to be consistent with a Weibull failure model of the glass under a stress field derived from the Eshelby inclusion model. Self-fractured spherulite failure was found to be consistent with a failure model based on thin films under biaxial stress.
\end{abstract}

\section{Introduction}

$\mathrm{F}$ OR over a century it has been understood that that crystallization to cristobalite weakens glass. The earliest reports of the crystallization of amorphous silica to cristobalite come from scientists frustrated by the clouding and subsequent breakage of fused-quartz lab-ware exposed to extreme temperatures, such as reported by Crookes in 1912. ${ }^{1}$ We know this weakening effect is a result of extensive microfracture upon the transition of cristobalite from the hightemperature beta-phase to the low-temperature alpha-phase at $\sim 250^{\circ} \mathrm{C} .^{2-4}$ This micro-fracture is caused by the $4.9 \%$ volume reduction on transition from alpha-to-beta. ${ }^{5}$

The development of stress in glass-ceramics has been widely reported upon in the literature; however, the work tends to be directed toward bulk-nucleated glass-ceramics in which the multiphase glass-ceramic can be used as a stiff load bearing material. ${ }^{6-9}$ Less well studied is the development of stress and failure in surface nucleating systems. This is likely because crystallization in such systems is considered a flaw rather than attribute. To our knowledge, no one has looked specifically at the stress development in the amorphous silicacristobalite system.

Crystallization in cristobalite occurs through the formation of surface spherulites which then grow and impinge to form

L. Pinckney-contributing editor a fully crystalline surface over an amorphous core. The lateral growth across the surface has been reported to occur faster than growth in thickness. ${ }^{10}$ During crystallization the glass matrix is above the annealing temperature so no stress develops. On cooling small thermal mismatch stresses can develop between the beta-cristobalite and amorphous silica. Although beta-cristobalite has very low thermal expansion, the thermal expansion of amorphous silica is nearly zero. On cooling from the strain temperature of $\sim 1100^{\circ} \mathrm{C}-300^{\circ} \mathrm{C}$ betacristobalite will experience a volume shrinkage of $\sim 0.58 \%$ relative to the amorphous silica. ${ }^{11}$ Upon cooling to $\sim 250^{\circ} \mathrm{C}$ beta-cristobalite transforms to alpha-cristobalite, resulting in a $4.9 \%$ volume reduction and the development of stress between the cristobalite and the amorphous matrix. ${ }^{5}$ Therefore, the transformation volumetric strain is $\sim 8.4$ times larger than the thermal expansion volumetric strain mismatch. In this work we make the approximation of only considering the much larger transformation strain. In the case of isolated spherulites, the beta-cristobalite develops stress free at high temperature, but on transformation to alpha-cristobalite the spherulite shrinks away from the matrix as shown schematically in Fig. 1.

In the present study the stress state of isolated spherulites and resulting fracture are observed. The fracture is modeled to understand the relationship between spherulite size and mode of fracture.

\section{Procedure}

\section{(1) Materials}

A high purity bulk silica glass was used in this study. GE 124 (GE Momentive, Willoughby, OH) glass prepared as $75 \mathrm{~mm} \times 25 \mathrm{~mm} \times 2.5 \mathrm{~mm}$ slides were used as the starting material. The purity of this material is greater than $99.9 \%$. The primary contaminants are aluminum with $14 \mathrm{ppm}$, titanium with $1.1 \mathrm{ppm}$, and hydroxyl with $<5 \mathrm{ppm}$. All other contaminants amount to $<4.5 \mathrm{ppm}$ in total. It should be noted that this is a very low hydroxyl content that reflects the material as-received material. Heating under ambient conditions will increase the hydroxyl content through absorption of atmospheric $\mathrm{H}_{2} \mathrm{O} \cdot{ }^{12,13}$ This will result in higher hydroxyl content in the material at crystallization.

\section{(2) Annealing and Observation}

GE 124 slides were annealed at $1200^{\circ} \mathrm{C}-1400^{\circ} \mathrm{C}$ with hold times ranging from $10 \mathrm{~min}$ to $6 \mathrm{~h}$. Annealing was conducted under ambient atmosphere with laboratory relative humidity ranging from $15 \%$ to $60 \%$. During annealing the slides were held on an alumina plate and supported by alumina spacers at four points near each corner. The slide was supported in this way to minimize contact contamination that may impact crystallization. ${ }^{13,14}$ The extent of beta-cristobalite formation, including the size and area fraction of the spherulites, did not change systematically with the annealing time and temperature. Instead the behavior was quite variable from one specimen to the next and from one location to another on
Manuscript No. 35005. Received May 14, 2014; approved July 24, 2014

* Member, The American Ceramic Society.

**Fellow, The American Ceramic Society.

†Author to whom correspondence should be addressed. e-mail: breneman@umich.edu 


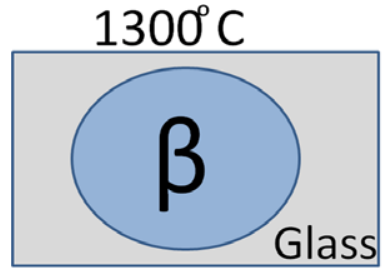

\section{Nucleation: $\mathrm{T}>\mathrm{Tg}$ spherulites grow in stress free matrix}

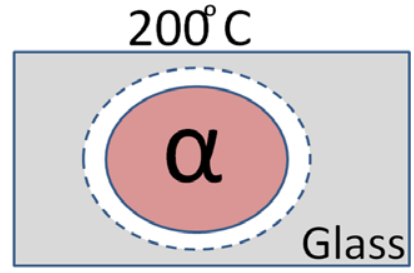

4.9 vol\% contraction of spherulite due to $\beta-\alpha$ transformation greatly increases tensile stress

Fig. 1. Illustration of the beta-to-alpha transformation for an isolated spherulite in a glass matrix. The $4.9 \%$ volume decrease on transformation to alpha from beta on cooling causes the spherulite to try to pull away from matrix inducing stress and/or failure.

the same specimen. As the apparent kinetics of cristobalite formation was so variable, we focus instead on the regular and systematic behavior of isolated spherulite after transformation to alpha-cristobalite.

Following annealing, samples were examined by optical microscopy. Polarized light was used in conjunction with a Nomarski prism to achieve contrast between the cristobalite and the amorphous silica matrix. The stress fields surrounding spherulites were qualitatively examined using crossedpolarizers optical microscopy (CPLOM).

\section{Results}

Spherulites were observed to surface nucleate and grow spherulitically as has been reported by others., ${ }^{3,15}$ Though often indistinct when spherulite symmetry can be determined it appears to be 3 -fold about the center of the spherulite. This indicates the cubic beta-cristobalite grew with a $<111>$ orientation. This agrees with the results of Presser et al. who found the orientation to be $\langle 111\rangle$ and growth to occur on the octahedral faces. ${ }^{15}$ When observed at room temperature, spherulites are in the tetragonal alpha-cristobalite phase having transformed from cubic beta-cristobalite. This alpha-cristobalite is highly twinned as a result of the loss of symmetry through the displacive beta-alpha transformation and also under stress due to the $4.9 \%$ volume reduction associated with the transformation.

While many spherulites remain intact some were observed to fracture within the spherulite while others were observed with encircling cracks in the glass matrix. A typical image with fractured and unfractured spherulites in the amorphous matrix can be seen in Fig. 2. An example of an encircling glass matrix crack and a close up of a fractured spherulite can be seen in Fig. 3. Amorphous silica is birefringent under stress. A spherulite surrounded by a radial stress field will polarize transmitted light to an orientation parallel to the stress. In crossed-polarizers optical microscopy (CPLOM), two polarizers oriented 90 degrees apart are utilized, one above and one below the sample. If the sample does not induce any polarization, the light that passes the first polarizer will be extinguished upon reaching the second due to the misorientaion of 90 degrees. In the case of a radial tensile field the light passing through the sample will reorient to be parallel to the stress field. The reoriented light passes through the second polarizer resulting in an illumination effect. In our case of a radial stress field the light will also be polarized. This radial polarization effect in the stressed amorphous silica results in an illumination effect with intensity that scales with the degree of misorientation relative to the polarizers. In the case of a radial stress field (such as that surrounding an alpha-spherulite) the observed intensity are at a maxima at $45^{\circ}$ misorientation to the polarizers. This results in a "Maltese Cross" pattern centered on the middle of the

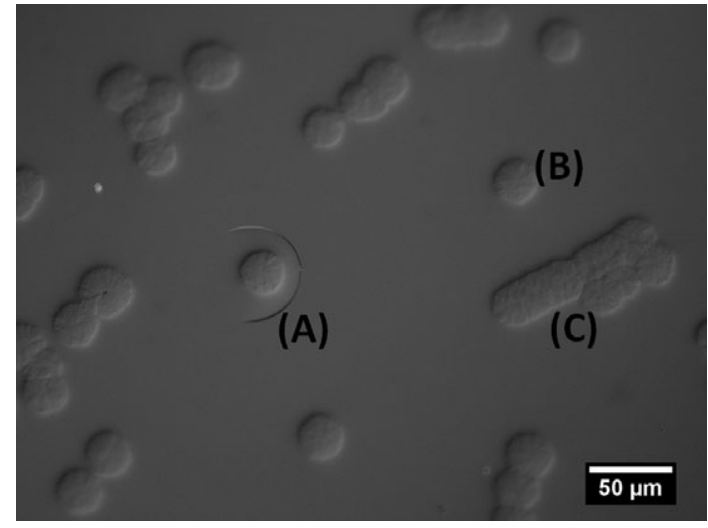

Fig. 2. Isolated spherulites in amorphous silica matrix viewed by reflected polarized light. Spherulites can be observed in both fractured and unfractured state. Sample was annealed at $1375^{\circ} \mathrm{C}$ for 2 h. (A) Isolated spherulite with encircling matrix crack. (B) Isolated spherulite surrounded by un-cracked matrix. (C) Impinged spherulites.

radial stress field. Conversely the misorientation and intensity are at a minimum when aligned with one polarizer or the other. The end results for polarizers oriented vertically and horizontally are intensity maxima at $45^{\circ}, 135^{\circ}, 225^{\circ}$, and $315^{\circ}$ and minima at $0^{\circ}, 90^{\circ}, 180^{\circ}$, and $270^{\circ}$.

The stress fields surrounding the alpha-cristobalite spherulites were imaged qualitatively by CPLOM, as seen in Fig. 3. An image with three neighboring spherulites imaged both by reflected polarized light and by cross-polarized light can be seen in Fig. 4. As discussed in the previous section, the intensity of a radially symmetric field has maxima at $45^{\circ}, 135^{\circ}$, $225^{\circ}$, and $315^{\circ}$ and minima at $0^{\circ}, 90^{\circ}, 180^{\circ}$, and $270^{\circ}$. In Fig. 4 the spherulites are oriented diagonally relative to the polarizers to maximize the intensity in between them. The stress concentration can be seen to be very intense in between the spherulites. In Fig. 5 an S-crack can be seen, likely initiating from the stress concentration between two adjacent spherulites.

At room-temperature spherulites were found to exist in one of three states: (1) Intact, with intact spherulites surrounded by intact but stressed glass matrix; (2) Matrix Cracked, with intact spherulites surrounded by an encircling crack passing through the glass matrix; (3) Self-Fracture, with "mud-crack" self-fracture of the cristobalite spherulite itself. These three states were found to coexist in the same sample. This can be seen in Fig. 6, in which examples of the three states can be seen in reflected polarized and transmitted cross-polarized imaging. A survey was conducted on the failure mode for isolated spherulites to look at the incidence of intact spherulites, matrix cracking, and spherulite self-fracture as a function of spherulite size. In total 2734 spherulites were observed from seven separate annealed samples. The results are presented as a cumulative plot as a function of size in Fig. 7 or spherulites 30 microns and larger; the spherulite size was recorded with an accuracy of \pm 5 microns. For spherulites smaller than 30 microns, the accuracy is \pm 2 microns. It can be seen that no fracture of the cristobalite or encircling cracks in the matrix occur for spherulites less than 15 microns in diameter. For intermediate sizes, some spherulites have matrix cracked with an encircling crack through the glass matrix. Spherulites 70 microns and greater in diameter are almost all self-fractured through the cristobalite in a "dry mud" manner. This cracking pattern is similar to the pattern of fracture seen when spherulites impinge creating a fully crystalline surface as seen in Fig. 8. Unlike the encircling cracks in the matrix seen in intermediate sized spherulites the "mud-cracking" self-fracture seems to occur in almost all spherulites above a certain size, this is illustrated in Fig. 9. The occurrence of both matrix cracks in the glass 


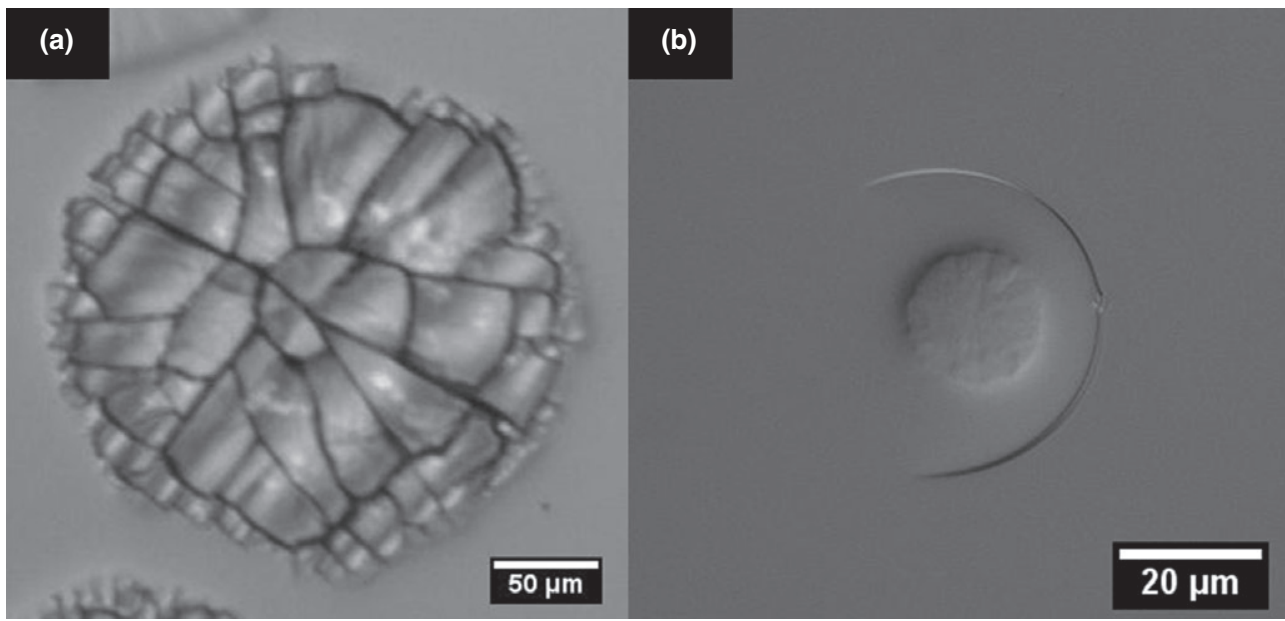

Fig. 3. Close up images of fractured spherulite and spherulite with a surrounding matrix crack viewed by reflected polarized light. Sample was annealed at $1375^{\circ} \mathrm{C}$ for $2 \mathrm{~h}$ a) fractured spherulite illustrating "mud cracking" b) spherulite with encircling crack passing through glass matrix.

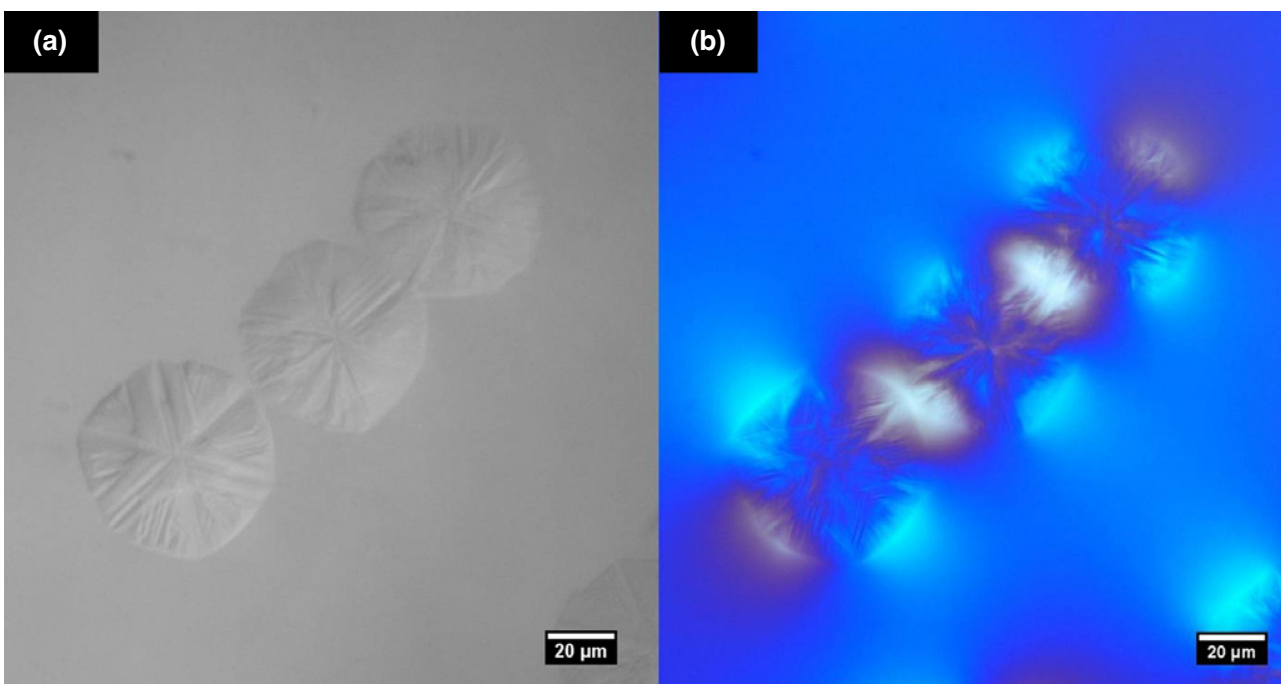

Fig. 4. Spherulites imaged under polarized reflected light (a) and imaged by cross-polarized transmitted light (b). Cross-polarized imaging highlights the stress concentration between spherulites. Sample was annealed at $1340^{\circ} \mathrm{C}$ for $40 \mathrm{~min}$.

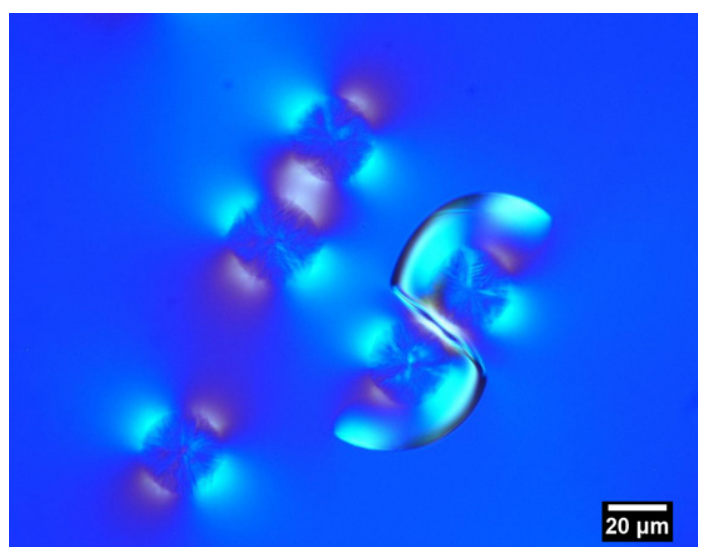

Fig. 5. Transmitted cross-polarized light image illustrating stress fields and matrix cracking. Sample was annealed at $1340^{\circ} \mathrm{C}$ for $40 \mathrm{~min}$.

and spherulite self-fracture of the cristobalite appear to be size dependent. However, matrix cracking was always a minority of the spherulites in a given sample; whereas above 70 microns almost all spherulites were observed to be self- fractured. This suggests that while both behaviors are size dependent they may depend on size in a different manner.

\section{Discussion}

\section{(1) Observed Failure Modes}

As seen in Fig. 6, following the beta-alpha transition, isolated spherulites were observed in one of three states: intact, matrix cracked, or self-fractured. Intact here refers to intact spherulites in intact matrix, while matrix cracked refers to intact spherulites encircled by a crack through the surrounding glass matrix. Self-fractured refers to spherulites in which the cristobalite has fractured. No spherulites were observed to have matrix cracks and also be self-fractured. These two different failure modes both appear to relieve stress, but also appear to be separate phenomena. Both failure modes seem to be size dependent with the population occurring at different spherulite diameters as seen in Fig. 7. Small spherulites with diameters 15 microns and smaller are all intact, displaying no cracks in the matrix or fracture of the spherulite. Spherulites between 15 and 70 microns in diameter sometimes are encircled by a crack through the matrix, with frequency of matrix cracking increasing with spherulite diameter. Spherulites above 70 microns in diameter self-fracture 


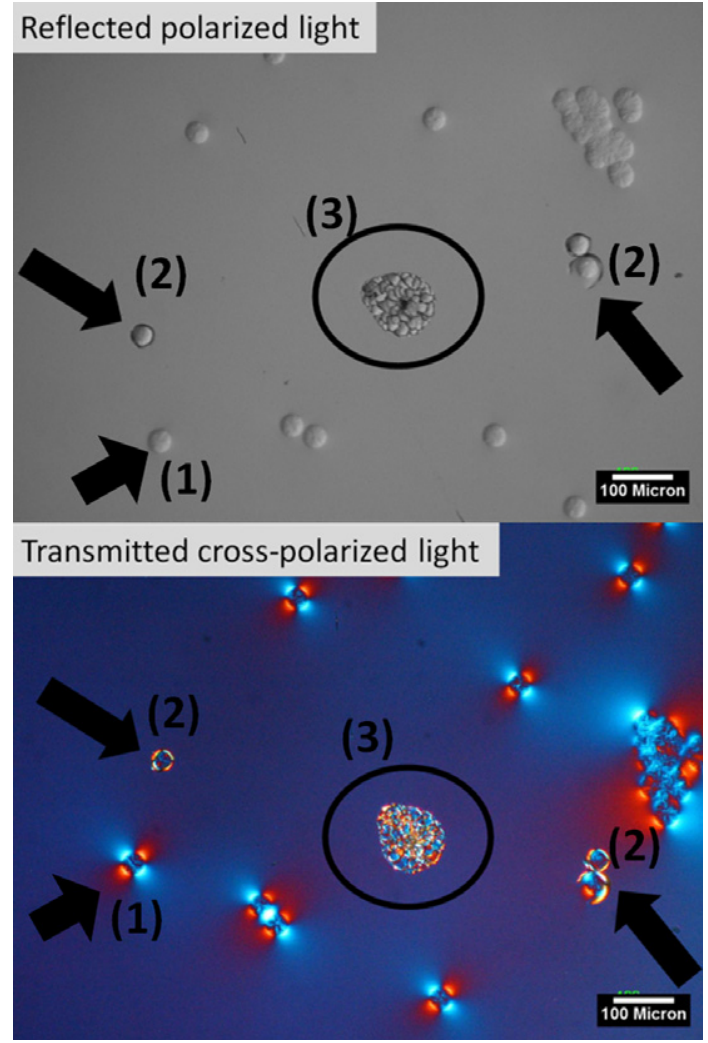

Fig. 6. Reflected polarized and crossed-polarized light images showing three behaviors of spherulites: (1) Stressed by no fracture; (2) Matrix cracking around spherulite; and (3) Fracture of the spherulite. Sample was annealed at $1340^{\circ} \mathrm{C}$ for $40 \mathrm{~min}$.

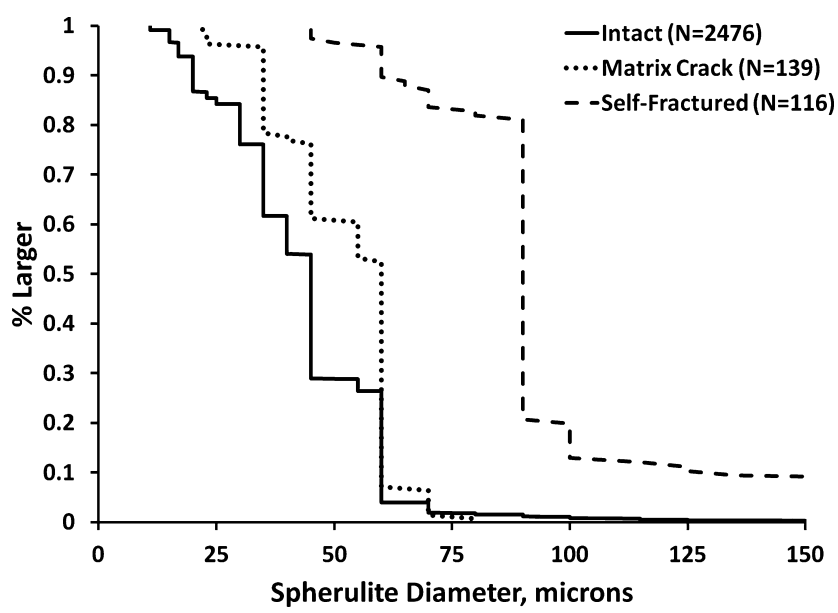

Fig. 7. Cumulative distribution by size of isolated spherulites for each failure behavior.

through the cristobalite, with very few exceptions. There were many more small intact spherulites than large spherulites with cracks. Figure 7 has the size distribution for 2479 intact spherulites, 139 larger spherulites with cracks in the glass matrix, and 116 large spherulites which had self-fractured. In the following sections, the stress distribution and the mechanics of the matrix cracking and spherulite self-fracture are modeled.

\section{(2) Stress Distribution Surrounding Alpha-Cristobalite Spherulites}

To understand why the behavior depends on the size of the spherulite, we model the stress distribution. The spherulite can be considered a hemispherical inclusion on the surface

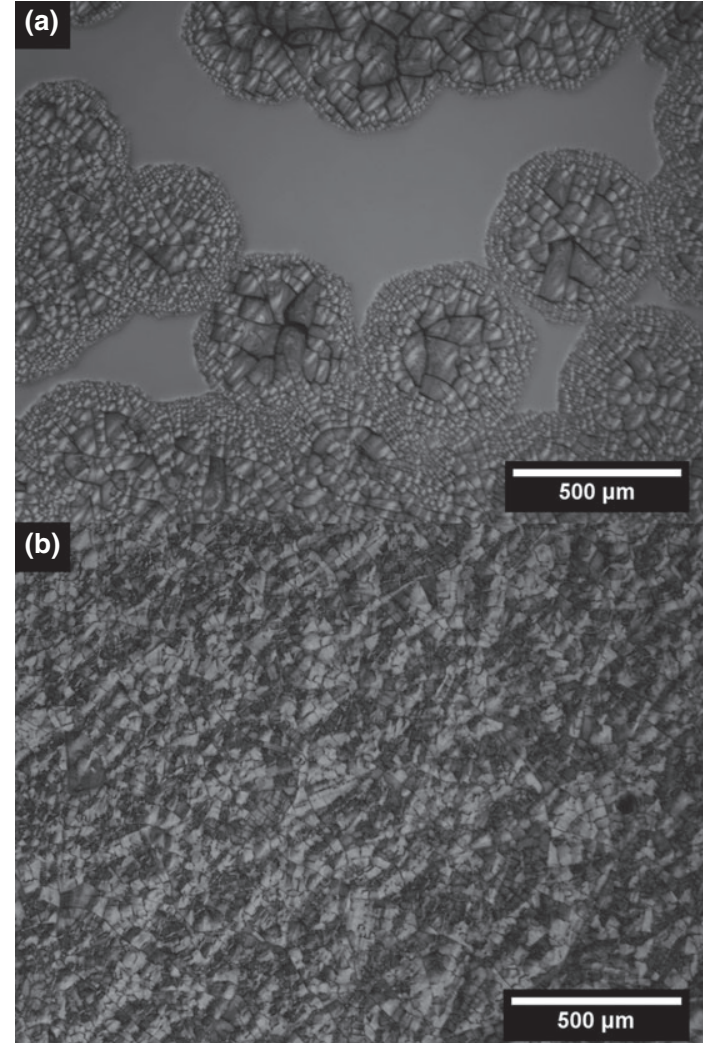

Fig. 8. Fractured surface of sample in which spherulites have begun impinging (a) and in which spherulites have all impinged (b) producing fully crystalline surface over an amorphous bulk. Sample was annealed at $1335^{\circ} \mathrm{C}$ for $40 \mathrm{~min}$.

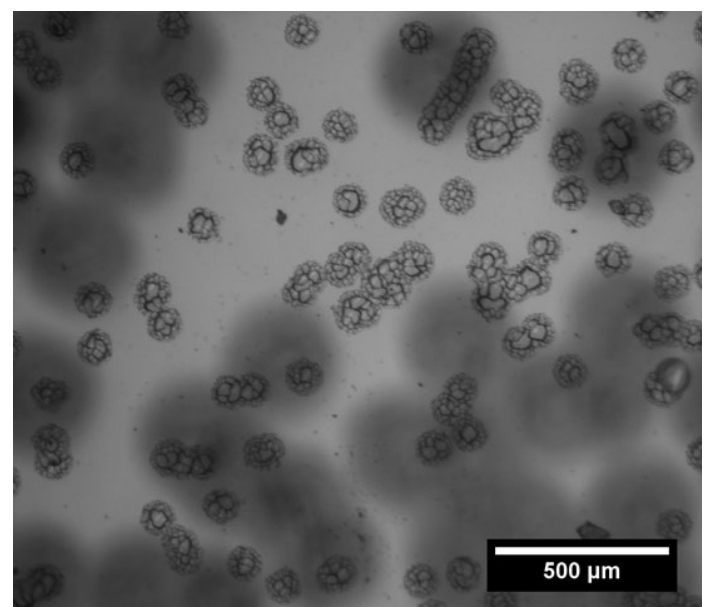

Fig. 9. A field of view with many spherulites of $\sim 90$ microns in diameter, all of which have self-fractured. The blurry dark background shapes are crystallization on the far surface. Sample was annealed at $1325^{\circ} \mathrm{C}$ for $40 \mathrm{~min}$.

of the glass. The well-known Eshelby model for a spherical inclusion can then be used to approximate the stress state in the surrounding matrix. In the classic Eshelby solution a spherical particle becomes strained relative to the matrix due to a thermal expansion mismatch. This strain leads to the development of a hydrostatic internal pressure and stress in the surrounding matrix. In the case of the spherulite in the matrix a transformation strain causes the inclusion (spherulite) to shrink relative to the matrix. This is analogous to the Eshelby treatment of stress development on cooling for an inclusion with a greater thermal expansion than the matrix. From the Eshelby solution in this case, the stress inside the 
inclusion is uniform tension, which is expressed as a pressure $p$, where: ${ }^{17}$

$$
p=\frac{2 E\left(\alpha_{\mathrm{m}}-\alpha_{p}\right)(\Delta T)}{(3-3 v)}=\sigma_{\mathrm{r}}^{(p)}=-\sigma_{\theta}^{(p)}=-\sigma_{\phi}^{(p)}
$$

Here $p$ is the internal Eshelby pressure, $E$ is the elastic modulus, $\alpha$ refers to the coefficient of thermal expansion of the matrix and inclusion respectively, and $\Delta T$ is the change in temperature. The three stresses on the right-hand side are the radial, angular, and azimuthal stress components in spherical coordinates. These stress components are all equal since inside the particle there is hydrostatic tension. Notice that the stress inside the particle is independent of size of the inclusion in this model.

For the case of the beta-to-alpha phase transformation of cristobalite spherulites in a glass matrix, the thermal expansion mismatch strain in Eq. (1) for the Eshelby form is replaced by the transformation strain, $\varepsilon_{\text {trans }}$ :

$$
p=\frac{2 E \varepsilon_{\text {trans }}}{(3-3 v)}=\sigma_{\mathrm{r}}^{(p)}=-\sigma_{\theta}^{(p)}=-\sigma_{\phi}^{(p)}
$$

Similarly the matrix stresses can be modeled. The stress in the glass matrix surrounding the spherulite has radial tension, and angular and azimuthal compression. Radial tension in the surrounding glass matrix damps with distance as $1 / r^{3}$ and increases with the cube of the particle size $a^{3}$ :

$$
\sigma_{\mathrm{r}}^{(m)}=\frac{2 E \varepsilon_{\text {trans }}}{(3-3 v)} \frac{a^{3}}{r^{3}}=p \frac{a^{3}}{r^{3}}
$$

This radial tension has the potential to cause the glass matrix to crack. The plane of the crack will be any concentric shell in the glass matrix around the spherulite. There also are compressive stresses in the angular and azimuthal directions. These are:

$$
\sigma_{\theta}^{(m)}=\sigma_{\phi}^{(m)}=\frac{-2 E \varepsilon_{\text {trans }}}{(3-3 v)} \frac{a^{3}}{r^{3}}=-p \frac{a^{3}}{r^{3}}
$$

These compressive forces are constant with the angles $\theta, \phi$, and damp out in magnitude with the square of the radial distance from the spherulite. They will not cause fracture, but they will suppress any angular or azimuthal component to the crack path. Therefore, a matrix crack will tend to circle around the spherulite. This model could explain why cracks initiated some distance from the spherulite encircle but do not approach the spherulite.

\section{(3) Mechanics of Glass Matrix Fracture}

Many spherulites have encircling cracks through the matrix. Such cracks could be caused by the tensile field surrounding the spherulite initiating cracking in the glass. The encircling cracks can occur some distance from the edge of the spherulite. This may be due to the cracks initiating from flaws in the glass, as often observed for the fracture of glass. This would imply that an interaction between the stress field intensity and the flaw distribution of the amorphous silica leads to the observed spacing between the spherulite and the encircling crack.

The highest magnitude of the matrix tension occurs at $r=a$, the boundary between the spherulite and the matrix. However, for an incremental spherical shell outside the spherulite, while the stress decreases as $1 / r^{3}$, the volume under stress increases as $4 \pi / 3\left(r^{3}-a^{3}\right)$. So in the region where the stress is highest, the stressed volume is smallest. Fracture in glass is often determined by the largest flaw found in the stressed volume, so the problem can be considered in the context of Weibull statistics.
The probability, $F$, of initiating an encircling matrix crack can be described as:

$$
F=1-\exp \left[\int_{a}^{\infty}-\left(\frac{\sigma(r)}{\sigma_{\mathrm{o}}}\right)^{m} \frac{V(\mathrm{r})}{V_{\mathrm{o}}} \mathrm{d} r\right]
$$

where $\sigma_{\mathrm{o}}$ and $\mathrm{m}$ are the characteristic strength and Weibull modulus for a two-parameter Weibull, and $V_{\mathrm{o}}$ is the reference volume associated with the characteristic strength. Since the stress and stressed volume change with distance $r$, we assess the failure probability integrated for all $r$ greater than the spherulite radius $a$. Equation (5) models how the probability of failure varies with stressed volume $V(r)$. Substituting values for the stress and volume terms allows the integration as follows:

$$
F=1-\exp \left[\int_{a}^{\infty}-\left(\frac{p a^{3}}{\sigma_{\mathrm{o}} r^{3}}\right)^{m} \frac{2 \pi r^{2}}{V_{\mathrm{o}}} \mathrm{d} r\right]
$$

Followed by:

$$
F=1-\exp \left[\frac{2 \pi}{V_{\mathrm{o}}}\left(\frac{p a^{3}}{\sigma_{\mathrm{o}}}\right)^{m} \int_{a}^{\infty} r^{2-3 m} \mathrm{~d} r\right]
$$

And finally:

$$
F=1-\exp \left[\frac{2 \pi}{V_{\mathrm{o}}}\left(\frac{p}{\sigma_{\mathrm{o}}}\right)^{m} \frac{a^{3}}{3 m-3}\right]
$$

So from Eq. (8), the probability of crack initiation in the matrix surrounding a single spherulite is simply a function of the Weibull parameters, the internal pressure $p$ derived from the Eshelby approximation, and the spherulite size, $a$. If we consider the size when the probability of matrix cracking is a particular value, say $37 \%$ (i.e., 1/e) to simplify the exponential, we have a critical spherulite size is $\mathrm{a}_{F=0.37}$ where:

$$
a_{F=0.37}=\left(\frac{[3 m-3] V_{\mathrm{o}}}{2 \pi}\right)^{\frac{1}{3}}\left(\frac{\sigma_{\mathrm{o}}}{p}\right)^{\frac{m}{3}}
$$

Thus, the likelihood of matrix cracking should increase strongly with the spherulite size, but remains a statistical phenomenon. This seems to match the behavior seen in Fig. 7.

Glass matrix cracking caused by spherulites is clearly related to size with larger sizes being associated with higher rates of matrix cracking; however, matrix cracking is always observed to coexist with unfractured spherulites. The Weibull model suggests that as spherulites get larger the probability of activating a flaw and triggering a matrix crack increases, but it remains a statistical phenomenon dependent on the Weibull parameters of the glass. Thus, the observed matrix cracks behave in the manner of a classic brittle failure induced by a stress field than can be approximated by Eshelby methods.

\section{(4) Mechanics of Spherulite Self-Fracture of Alpha- Cristobalite}

The self-fractured spherulites can be considered separately from glass matrix cracking, because the fracture occurs within the cristobalite and the crack morphology differs. The geometry of the spherulites may also change as they grow as others have reported faster growth laterally than in depth; ${ }^{10}$ however, we were unable to accurately measure depth of the spherulites and so cannot confirm this. Self-fractured spherulites exhibit a cracking pattern reminiscent of dried mud. This pattern of cracking is not uncommon in thin-film applications. In many 
thin-film applications a stress develops between the film and the substrate, such as that due to a mismatch in thermal expansion. Often such films develop channel cracks to alleviate this stress, resulting in dried-mud morphology. This may suggest the largest spherulites are behaving similar to thin films under stress. In fact, as can be seen in Fig. 8 when the spherulites impinge and create a fully crystalline surface the fracture pattern remains similar to that of self-fractured spherulites. This supports the hypothesis that self-fractured spherulites are behaving like thin films. A cristobalite layer on an amorphous silica matrix will attempt to shrink on passing the beta-toalpha transition, but it will be constrained by the underlying matrix. The constraint from the underlying glass results in biaxial stresses. This situation is directly analogous to a thin film on a thick substrate experiencing biaxial tension.

A thin film under biaxial stress on an infinite matrix is a well-defined system. In such systems the stress often arises from a strain resulting from the thermal expansion mismatch between the film and substrate. In the case of the spherulites the strain is the strain from the beta-to-alpha transition. Cristobalite and amorphous silica both have an elastic modulus of $72 \mathrm{GPa}$ which further simplifies the relationship. The fracture of thin films on infinite substrates with identical elastic moduli is governed by:

$$
\frac{\left(E /\left(1-v^{2}\right)\right) G_{\mathrm{ss}}}{\sigma_{\mathrm{o}}^{2} h}=1.98
$$

in which $G_{\mathrm{ss}}$ is the steady-state energy release due to fracture. ${ }^{18}$ Fracture is expected if $G_{\mathrm{ss}} \geq \Gamma$, where $\Gamma$ is the cohesive energy of the material. This provides a critical thickness, $h_{\mathrm{c}}$, for failure given transformation strain, $\varepsilon_{\text {trans }}$ as follows:

$$
\frac{\Gamma}{\left(E /\left(1-v^{2}\right)\right) \varepsilon_{\text {trans }}^{2} 1.98}=h_{c}
$$

For a given strain energy, elastic modulus, Poisson's ratio, and cohesive energy there is a critical film thickness at which the film cracks spontaneously. One can suppose that for spherulites the thickness, $h$, is a function of the diameter, $a$. If we make an approximation that $a=b^{*} h$ we can rewrite Eq. (11) in terms of spherulite diameter:

$$
\frac{b_{\Gamma}}{\left(E /\left(1-v^{2}\right)\right) \varepsilon_{\text {trans }}^{2} 1.98}=a_{c}
$$

This suggests that above a critical diameter the spherulite is expected to be able to lower its energy by spontaneously fracturing. This matches the behavior seen for spherulite fracture. Above a certain size the spherulites are observed to nearly universally self-fracture. A field of spherulites above this critical size can be seen in Fig. 9. The critical value for spherulite diameter, $a_{c}$, appears to be $\sim 70$ microns as very few spherulites are unfractured above that size. It seems that self-fractured spherulites are exhibiting fracture behavior similar to thin films. Upon reaching a certain size the strain energy associated with the biaxial constraint of the transformation strain induces fracture of the spherulite.

\section{Conclusions}

Cristobalite spherulites were formed on the surface of fully dense amorphous silica and allowed to pass the beta-to-alpha transition upon cooling. Stress fields were observed to surround unfractured samples by crossed-polar microscopy. Fractured spherulites were found to have failed by one of two mechanisms, either by encircling cracks passing through the matrix or "mud-cracking" of the spherulite itself. Matrix cracks were observed to always coexist with unfractured spherulites, and the frequency of cracking was observed to increase with spherulite size. Spherulite fracture on the other hand, was observed in larger spherulites, and nearly all spherulites above 70 microns in diameter were self-fractured. Applying a Weibull statistics to glass cracks induced by an Eshelby stress field produced a model for matrix cracking. This model predicts probabilistic matrix hoop cracking with a dependence on spherulite size, which aligns with observation. The occurrence of matrix cracks is a size dependent but statistical phenomenon. A separate model based on thin-film mechanics was formulated for spherulite fracture. The spherulite behaves as a thin film under biaxial stress over an infinite matrix. This model predicts a critical size at which the spherulite will fracture. This critical size matches the observation that above a certain size, nearly all spherulites have self-fractured. Based on the models and observations, a complete picture of failure can be developed. Upon transitioning from beta-alpha stress is induced by the $\sim 5 \%$ volume reduction. In spherulites above a critical size this results in spontaneous self-fracture. In spherulites below the critical size, the stress field may induce cracking if the stress field activates a sufficiently large flaw. Larger spherulites induce larger matrix stresses over larger areas increasing their probability of activating a flaw, but matrix cracking remains a statistical phenomena.

\section{Acknowledgments}

This research was supported by the Defense Advance Research Projects Agency (DARPA) under grant no. HR001-08-1-0075, Principal Investigator Suman Das, Georgia Institute of Technology, Program Officer W.S. Coblenz and the Office of Naval Research, Scientific Officer David Shifler. We thank Professor Michael Thouless for helpful comments on the mechanics.

\section{References}

${ }^{1}$ W. Crookes, "On the Devitrification of Silica Glass," Proc. R. soc. Lond. Ser. A-Contain. Pap. Math. Phys. Character, 86 [588] 406-8 (1912).

${ }^{2}$ R. B. Sosman, The Phases of Silica. Rutgers University Press, New Brunswick, NJ, 1965.

${ }^{3}$ N. G. Ainslie, C. R. Morelock, and D. Turnbull, "Devitrification Kinetics of Fused Silica," in Symposium on Nucleation and Crystallization in Glasses and Melts.

${ }^{4}$ F. E. Wagstaff, "Crystallization Kinetics of Internally Nucleated Vitreous Silica,” J. Am. Ceram. Soc., 51 [8] 449-53 (1968).

${ }^{5}$ D. R. Peacor, "High-Temperature Single Crystal Study of Cristobalite Inversion," Z. Kristall, 138, 274-98 (1973).

${ }^{6}$ V. R. Mastelaro and E. D. Zanotto, "Residual Stresses in a Soda-Lime-Silica Glass-Ceramic," J. Non-Cryst. Solids, 194 [3] 297-304 (1996).

${ }^{7}$ O. Peitl, F. C. Serbena, V. R. Mastelaro, and E. D. Zanotto, "Internal Residual Stress Measurements in a Bioactive Glass-Ceramic Using Vickers Indentation," J. Am. Ceram. Soc., 93 [8] 2359-68 (2010).

${ }^{8}$ F. C. Serbena and E. D. Zanotto, "Internal Residual Stresses in GlassCeramics: A Review," J. Non-Cryst. Solids, 358 [6-7] 975-84 (2012).

${ }^{9}$ E. D. Zanotto, "Glass Crystallization Research A 36-Year Retrospective. Part I, Fundamental Studies,” Int. J. Appl. Glass Sci., 4 [2] 105-16 (2013).

${ }^{10}$ R. S. Hay, G. E. Fair, R. Bouffioux, E. Urban, J. Morrow, A. Hart, and M. Wilson, "Relationships Between Fiber Strength, Passive Oxidation, and Scale Crystalization Kinetics in HI-Nicalon-S SiC Fibers," pp. 39-51 in Mechanical Properties and Performance of Engineering Ceramics and Composites Vi, Vol. 32, Edited by D. Singh, J. Salem, and S. Widjaja, Ceramic Engineering and Science Proceedings, 2011.

${ }^{11}$ I. P. Swainson and M. T. Dove, "On the Thermal Expansion of Beta-Cristobalite," Phys. Chem. Miner., 22 [1] 61-5 (1995).

${ }^{12}$ T. Honma, N. Tamura, K. Saito, and E. Sekiya, "Difference in Structural Relaxation Times of Inner Surface and Inner Bulk Region of Silica Glass Arc Tube," New J. Glass Ceram., 3, 48-52 (2013).

${ }^{13}$ P. P. Bihuniak, "Effect of Trace Impurities on Devitrification of Vitreous Silica," J. Am. Ceram. Soc., 66 [10] C188-9 (1983).

${ }^{14}$ E. Opila, "Influence of Alumina Reaction Tube Impurities on the Oxidation of Chemically-Vapor-Deposited Silicon-Carbide," J. Am. Ceram. Soc., 78 [4] 1107-10 (1995).

${ }^{15}$ V. Presser, A. Loges, Y. Hemberger, and K. G. Nickel, "Microstructural Evolution of Silica on Single-Crystal Silicon Carbide. Part I: Devitrification and Oxidation Rates," J. Am. Ceram. Soc., 92 [3] 724-31 (2009).

${ }^{16}$ F. E. Wagstaff and K. J. Richards, "Preparation and Crystallization Behavior of Oxygen Deficient Vitreous Silica," J. Am. Ceram. Soc., 48 [7] 382-3 (1965)

${ }^{17}$ J. D. Eshelby, "The Determination of the Elastic Field of an Ellipsoidal Inclusion, and Related Problems," Proc. R. Soc. A-Math. Phys. Eng. Sci, 241 [1226] 376-96 (1957).

${ }^{18}$ M. S. Hu, M. D. Thouless, and A. G. Evans, "The Decohesion of ThinFilms From Brittle Substrates," Acta Metall., 36 [5] 1301-7 (1988). 